\title{
Changement climatique dans le bassin versant de l’Ogooué : évolution récente et impact sur les écoulements
}

\author{
Valentin Brice Ebodé ${ }^{1}$, Gil Mahé ${ }^{2}$, and Ernest Amoussou ${ }^{3}$ \\ ${ }^{1}$ Département de Géographie, Université de Yaoundé 1, Yaoundé, Cameroun \\ ${ }^{2}$ Institut de Recherche pour le Développement, Marseille, France \\ ${ }^{3}$ Département de Géographie et Aménagement du Territoire, Université de Parakou, Parakou, Bénin \\ Correspondence: Valentin Brice Ebodé (ebodebriso@gmail.com)
}

Published: 16 November 2021

\begin{abstract}
Résumé. L'objectif de cet article est de faire une évaluation des tendances récentes des grandeurs hydroclimatiques dans le bassin de l'Ogooué en contexte de changement climatique. Pour cela, les données de pluies et de débits de ce bassin ont été analysées au moyen du test de Pettitt. Les résultats de cette étude révèlent une diminution statistiquement significative des modules annuels que le test de Pettitt situe en 1972-1973, mais rien de tel pour les pluies à ce même pas de temps. Les écarts des moyennes décennales à la moyenne interannuelle montrent cependant des liens plus nets entre ces variables, se traduisant essentiellement par une diminution synchrone au cours des décennies 1970 et 1980 . Cette diminution est suivie d'une reprise au cours des deux décennies d'après (1990 et 2000), laquelle s'estompe à nouveau au cours de la décennie 2010. Les écoulements des saisons pluvieuses (printemps et automne) ont enregistré les modifications les plus importantes, suite aux variations des régimes pluviométriques des saisons sèches (hiver et été) qui les précèdent. La saison sèche d'hiver a connu entre les décennies 1970-1990 une diminution importante des précipitations qui a provoquée une chute des écoulements du printemps. Le rehaussement et la flexion respectivement notés au cours des décennies 2000 et 2010 s'accompagnent des mêmes tendances dans les écoulements du printemps. A l'inverse, entre les décennies 1980-1990, il a été noté une hausse conjointe des pluies d'été et des écoulements d'automne. La flexion des pluies d'été notée depuis la décennie 2000 est également perceptible dans les écoulements d'automne. Ces résultats pourraient servir à renforcer les capacités de gestion des ressources en eau dans le bassin versant concerné et dans la région. Ils apportent également de nouveaux éléments pour étudier et comprendre la variation saisonnière et la disponibilité de l'eau douce en aval, dans les estuaires et les zones côtières des rivières régionales.
\end{abstract}

The objective of this article is to assess recent trends of hydroclimatic quantities in Ogooue basin in the context of climate change. For this, the rainfall and discharges data of this basin were analyzed using the Pettitt test. The results of this study reveal a statistically significant decrease in runoff that the Pettitt test situates in 1972-1973, but nothing like that for rainfall at this same time scale. The decadal deviations from the interannual average, however show links between these variables essentially marked by a synchronous decrease in the 1970s and 1980s. This decrease is followed by a recovery in the two decades following (1990 and 2000), which stops again during the 2010s. The flow of the rainy seasons (Spring and Autumn) recorded the most important modifications, following variations in the rainfall regimes of the dry seasons (Winter and Summer) which precede them. The dry Winter season experienced a significant decrease in precipitation between the 1970s and 1990s, which caused a decrease in Spring flows. The increase and flexion noted respectively during the 2000 and 2010 decades are accompanied by the same trends in Spring flows. Conversely, between the 1980s and the 1990s, there was a joint increase in Summer rainfall and Autumn flows. The decrease of Summer rainfall noted since the 2000s is also noticeable in the Autumn flows. These results could be used to strengthen water 
resources management capacities in the watershed concerned and the region. They also provide new insights to study and understand seasonal variation and availability of freshwater downstream, in estuaries and coastal areas of regional rivers.

\section{Introduction}

L'intérêt désormais consacré à la compréhension de la variabilité climatique en Afrique centrale provient de son impact sur les écoulements, et ainsi sur les économies des pays en développement de cette région. L'Ogooué, l'un des plus grands fleuves d'Afrique centrale équatoriale par sa longueur (environ $1000 \mathrm{~km}$ ), constitue un énorme espoir de vitalité pour les populations des pays qu'il traverse. Prenant sa source au Congo-Brazzaville, dans la partie occidentale des plateaux Batékés, sur les monts Massa, il parcourt essentiellement le territoire gabonais avant de se jeter dans l'océan Atlantique. Ce fleuve a fait l'objet d'un nombre relativement modeste de pertinentes études tant hydrologiques que climatologiques depuis le début de ce vingtième siècle; et pour cause, le manque de données d'observation sur cette période. Le peu d'études existantes a porté aussi bien sur l'évaluation de la ressource en eau que sur des projets d'aménagements potentiels dans les domaines hydro-agricole et hydroélectrique ou encore sur la propagation des crues (E. D. F., 1961 ; Carré, 1978; Buisson, 1985a et b; Mahé et al., 1990, 2013; Bogning et al., 2020; Ebodé, 2020). La station de Lambaréné est parmi les plus anciennes et l'une des mieux étudiées sur ce cours d'eau. Les études sur cette station (Mahé et al., 1990, 2013; Bogning et al., 2020; Ebodé, 2020) ont déjà montré des changements climatiques avec des modifications du régime hydrologique du fleuve à partir de la décennie 1970. Bien qu'ayant déjà une certaine connaissance des modifications du régime hydrologique de l'Ogooué à cette station, il convient néanmoins de l'actualiser en intégrant les données des deux récentes décennies, compte tenu de ce que la quasi-totalité d'études sus-évoquées se fondent sur des données qui n'excèdent pas la décennie 1990. L'objectif principal de cet article est donc de documenter le type et l'ampleur des changements de régime hydrologique de l'Ogooué dus aux changements climatiques sur la période récente (1929-1930 à 2017-2018).

\section{Cadre, données et méthodes}

L'étude porte sur le bassin versant de l'Ogooué à Lambaréné $\left(215000 \mathrm{~km}^{2}\right)$. Ce bassin est situé en Afrique centrale, approximativement entre les latitudes $2^{\circ} 42^{\prime} \mathrm{N}$ et $3^{\circ} 10^{\prime} \mathrm{S}$ et les longitudes $10^{\circ} 9^{\prime} \mathrm{E}$ et $14^{\circ} 30^{\prime} \mathrm{E}$ (Fig. 1). Il appartient au domaine équatorial avec des précipitations annuelles abondantes, oscillant autour de $1800 \mathrm{~mm}$, réparties sur quatre saisons d'inégale importance, dont deux sèches et deux pluvieuses. Les saisons de pluies (printemps et automne) sont

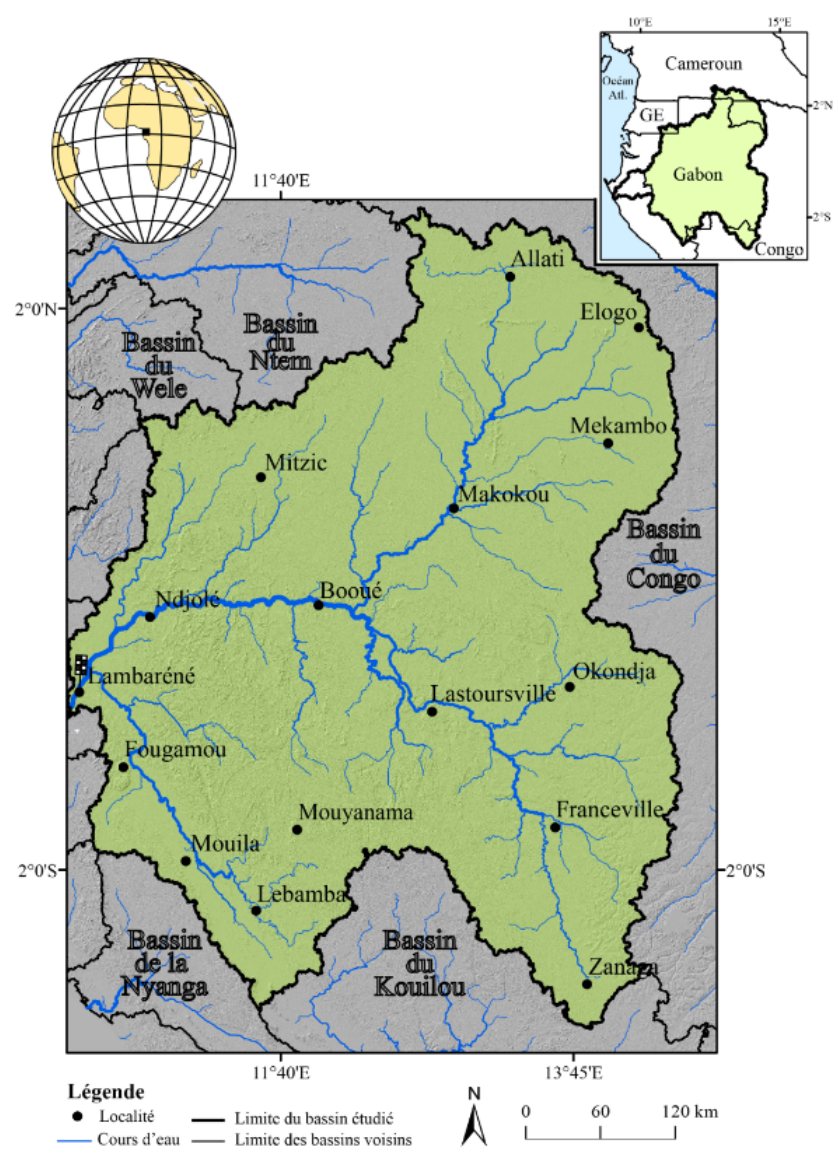

Figure 1. Localisation du bassin versant de l'Ogooué.

généralement très humides avec des totaux records avoisinant $800 \mathrm{~mm}$ et marquées par de nombreux orages qui occasionnent parfois des crues importantes. La saison sèche d'été est la seule véritable saison sèche de la région, avec des précipitations moyennes oscillant autour de $200 \mathrm{~mm}$; la saison sèche d'hiver étant considérablement humide dans la région, avec des pluies moyennes sensiblement deux fois plus importantes que celles d'été.

Les séries de précipitations et de débits ont été utilisées dans ce travail. Les débits (journaliers) de l'Ogooué proviennent du SIEREM (Système d'Informations Environnementales sur les Ressources en Eau et leur Modélisation). Elles contiennent des lacunes sur les intervalles 1950-1953 et 1989-2000. Les débits de base ont été extraits du débit total à partir du filtre récursif Aquapak, en téléchargement libre sur : https://www.jacobs.com/natural-resource-management (la date du dernier accès : 5 mai 2019). 
Les séries pluviométriques proviennent de deux sources. Sur la période 1940-1999, les grilles de pluies mensuelles SIEREM ont été utilisées. Leur unité d'espace est une maille de surface $0,5^{\circ} \times 0,5^{\circ}$, soit approximativement $2750 \mathrm{~km}^{2}$ dans la zone considérée (Dieulin et al., 2019). Ces grilles sont en accès libre aux formats ASCII et NetCDF sur le site http://www.hydrosciences.org/spip.phplunskiplpenaltyl @ M Mhskip.5\fontdimen2\font|relax?article1387 (la date du dernier accès : 7 mai 2019). Avant toute utilisation, il a fallu les évaluer d'abord au moyen des comparaisons avec les pluies issues des stations. Entre 2000 et 2017, le choix a été porté sur les estimations de pluies journalières TRMM 3B42 V7, remplacées elles-mêmes par les pluies GPM (Global Precipitation Measurement) depuis 2015. L'ensemble de ces estimations de pluies sont téléchargeables gratuitement sur le site https://giovanni.gsfc.nasa.gov (la date du dernier accès : 11 juin 2019), au format ASCII et à une résolution spatiale de $0,25^{\circ} \times 0,25^{\circ}$. Les cumuls mensuels des pluies TRMM et GPM calculés ont d'abord été validés au moyen des comparaisons avec les pluies issues des stations, puis avec les pluies SIEREM sur les années de disponibilité commune (1998 et 1999). Les coefficients de correction (essentiellement compris entre 1,34 et 1,65) ont par la suite été établis dans le cas des mois pour lesquels les écarts entre les deux sources (SIEREM et TRMM) étaient importants (juin et août).

L'analyse des pluies, des débits et des coefficients d'écoulement a été réalisée à l'aide de tests statistiques de détection de rupture (corrélation sur le rang et test de Pettitt; Lubès et al., 1994) au seuil de signification $95 \%$. Le test de Pettitt semble être le plus approprié pour l'analyse des séries lacunaires comme celles utilisées dans cette étude, parce qu'il sépare la série uniquement en deux périodes ayant un comportement globalement distinct, ce qui évite la détection des fausses ruptures comme on peut parfois l'observer avec d'autres tests comme la segmentation d'Hubert. Son choix au détriment des tests de tendance (test de Mann-Kendall par exemple) se justifie par le fait qu'il indique une date à partir de laquelle le changement devient significatif statistiquement parlant (rupture), ce qui permet une meilleure appréciation de la variabilité.

\section{Résultats et discussion dans le contexte régional}

\subsection{Evolution des pluies annuelles et saisonnières de l'Ogooué}

Les précipitations annuelles du bassin versant de l'Ogooué évoluent de manière non significative à la diminution entre 1940-1941 et 2017-2018 (Fig. 2). Le test de Pettit ne détecte d'ailleurs aucune rupture dans leur série chronologique. L'analyse des moyennes décennales révèle cependant dans leur évolution, une alternance des séquences sèches et humides plus ou moins longues. Ainsi, il a été observé au cours des décennies 1990 et 2000 une légère reprise consécutive à la sécheresse des décennies 1970 et 1980, qui semble avoir été interrompue à nouveau au cours de la décennie 2010 (Tableau 1). Ce retour à des conditions plus humides au cours de la décennie 1990 a également été suggéré partout en Afrique de l'ouest comme au Sénégal (Alhassane et al., 2013), au Burkina Faso (Lodoun et al., 2013) et au Niger (Ozer et al., 2009). Cependant, le rehaussement signalé dans cette étude s'estompe au cours de la décennie 2010, tandis que celui relevé en Afrique de l'ouest persiste jusqu'à présent (Descroix et al., 2020).

Les pluies des deux saisons sèches évoluent en tendances croisées sur le bassin versant de l'Ogooué (Fig. 2). Les cumuls de la saison sèche d'été évoluent statistiquement à la hausse entre 1940-1941 et 2017-2018. Le test de Pettit met en évidence dans leur série chronologique une rupture en 1979-1980 (Fig. 2). Le surplus par rapport à la moyenne enregistré suite à cette dernière est estimé à $+12,5 \%$. Pourtant excédentaire dans son ensemble, il est observé pour les précipitations de cette saison un relâchement progressif après la forte augmentation des décennies 1980 et 1990 . Ce relâchement devient davantage important au cours de la décennie 2010, pour laquelle il est noté un écart négatif par rapport à la moyenne interannuelle de $-3,2 \%$ (Tableau 1). Les pluies de la saison sèche d'hiver évoluent de manière non significative à la baisse. Cependant, on observe dans leur évolution une chute progressive entre les décennies 1970 et 1990, puis un relèvement au cours des deux décennies d'après, qui semble quand-même beaucoup plus important pour la décennie 2000 (Tableau 1).

Les cumuls des saisons pluvieuses (automne et printemps) n'ont pas connu de diminution significative d'après le test de Pettit. Les écarts des moyennes décennales à la moyenne interannuelle sont certes faibles dans l'ensemble, mais ils laissent transparaître comme dans le cas des totaux annuels, plusieurs séquences aux tendances évolutives différentes (Tableau 1). De l'analyse de ces écarts, il ressort que les pluies du printemps ont diminué de $2 \%$ et $4,5 \%$ au cours des décennies 1970 et 1980. Mais depuis le début de la décennie 1990, on note en général dans leur évolution un très léger rehaussement (Tableau 1). Dans le cas des pluies d'automne, une hausse de $+1,5 \%$ et $+4,3 \%$ a été enregistrée durant les décennies 1980 et 1990, suivie d'une faible diminution depuis la décennie 2000 .

\subsection{Evolution des modules annuels et saisonniers de l'Ogooué}

Les modules de l'Ogooué (annuels et saisonniers) évoluent à la baisse sur la période d'étude (Fig. 2). Cette diminution n'est pas significative statistiquement parlant dans le seul cas de l'automne (Fig. 2). Après la rupture observée en 1972-1973 dans les différents cas, les déficits par rapport à la moyenne interannuelle calculés pour les modules annuels, du printemps, de l'été et de l'hiver sont respectivement de $-6,4 \% ;-10,3 \% ;-6,9 \%$ et $-6,4 \%$ (Fig. 2). En dehors de l'automne, les écarts décennaux montrent une chute géné- 
Pluies

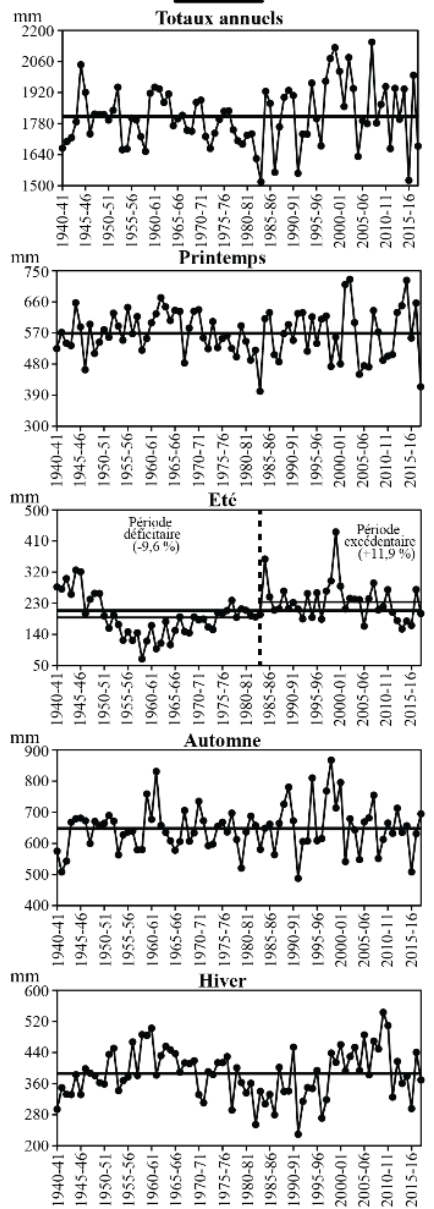

Débits
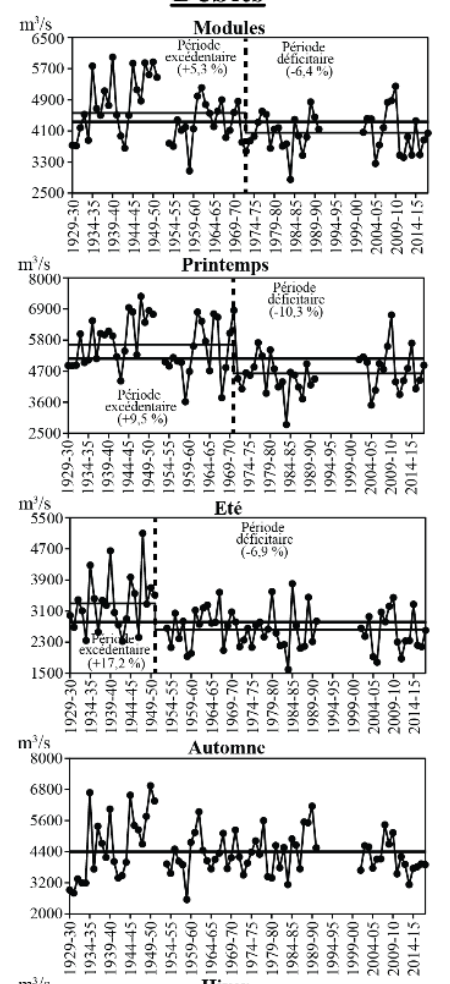
Hiver

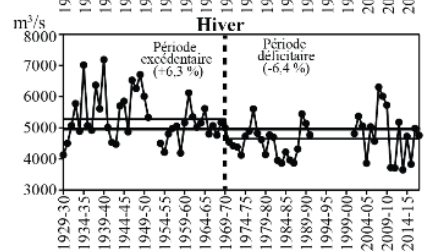

Débits de base

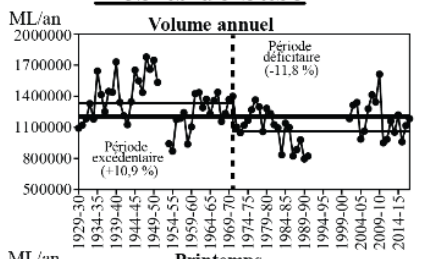

$\mathrm{ML} / \mathrm{an}=\frac{1}{210000}$

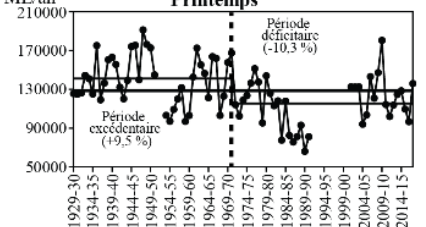

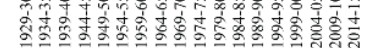
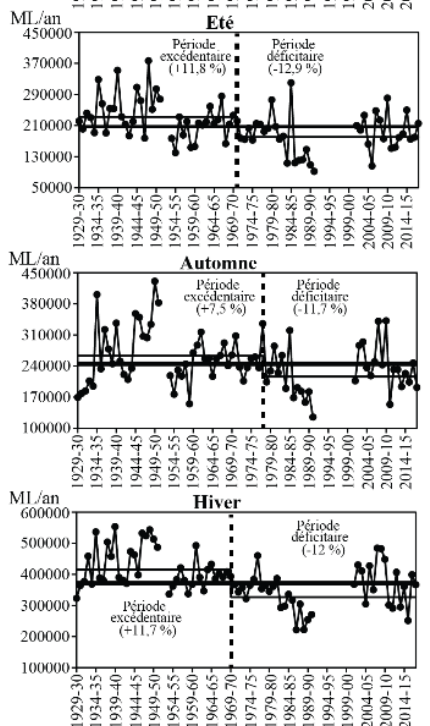

$\underline{\text { Ke }}$

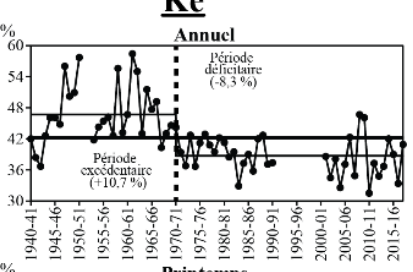

\%
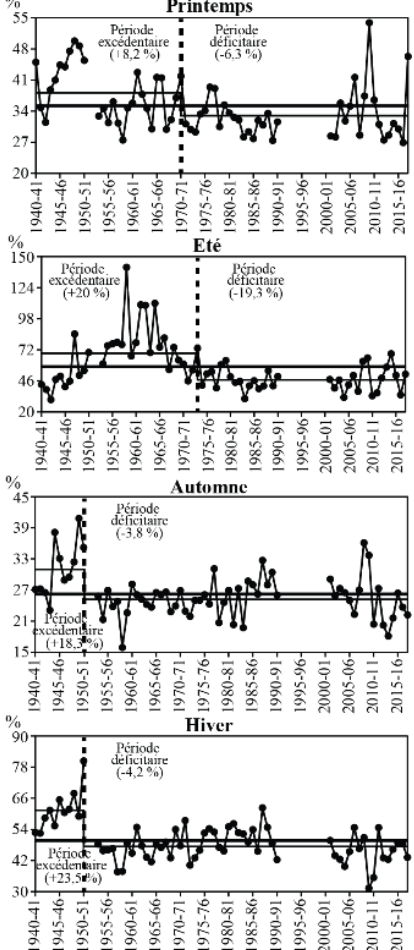

Figure 2. Evolution des pluies, débits, débits de base et coefficients d'écoulement annuels et saisonniers du bassin du l'Ogooué sur leurs périodes d'études respectives.

ralisée ayant débuté au cours de la décennie 1970, qui s'est considérablement amplifiée durant la décennie 2010. Cette décennie est la plus sèche de l'ensemble de la période d'observation (Tableau 1).

Les résultats de cette étude corroborent les conclusions des premières études menées dans la région. Cette étude confirme une rupture dans la série des modules annuels de l'Ogooué durant le décennie 1970 (en 1972-1973), ce qui est en accord avec les travaux de Liénou et al. (2008) et Conway et al. (2009) qui mettent en évidence des ruptures respectivement sur le Ntem en 1970 et l'Ogooué en 1977. Le présent travail relève également une diminution plus importante de la crue du printemps comparée à celle d'automne. Un constat identique a été fait par Mahé et al. (2013) sur ce même bas$\sin$.

\subsection{Sensibilité des écoulements aux variations interannuelles des précipitations}

Il est certes vrai que la stationnarité des pluies et des écoulements ne montre pas de liens apparents sur le bassin versant de l'Ogooué aux pas de temps étudiés (annuel et saisonnier), mais l'analyse des écarts décennaux à la moyenne interannuelle montre des liens relativement importants entre ces variables. Au pas de temps annuel, les tendances observées pour les précipitations entre les décennies 1970-1980 (baisse), 1990-2000 (rehaussement) et au cours de la décennie 2010 (rechute) sont identiques à celles enregistrées pour les écoulements (Tableau 1). On remarque cependant une diminution de débits plus forte que celle des pluies. Ceci ne pourrait s'expliquer que par une diminution des apports souterrains, ce que semble corroborer l'étude des débits de base. En effet, les débits de base diminuent considérablement, ce qui indique une vidange des nappes. Après les séries de sécheresse des années 1970 et 1980, on a assisté à un appauvrissement sévère des nappes phréatiques si bien que l'amé- 
Tableau 1. Ecarts (\%) des moyennes décennales annuelles et saisonnières des pluies, débits, débits de base et coefficients d'écoulement par rapport à leurs moyennes interannuelles sur le bassin étudié.

\begin{tabular}{|c|c|c|c|c|c|c|}
\hline \multirow[t]{2}{*}{ Variables } & \multirow[t]{2}{*}{ Périodes } & \multicolumn{5}{|c|}{ Ecarts à la moyenne interannuelle (\%) } \\
\hline & & Annuel & Printemps & Eté & Automne & Hiver \\
\hline \multirow{8}{*}{ Précipitations } & 1940-1949 & $-0,5$ & $-2,8$ & 29,6 & $-3,5$ & $-8,1$ \\
\hline & $1950-1959$ & $-1,7$ & 2,1 & $-31,1$ & $-1,1$ & 7,5 \\
\hline & $1960-1969$ & 1,7 & 7,5 & $-28,8$ & 0,9 & 11,1 \\
\hline & 1970-1979 & $-2,7$ & $-1,9$ & $-7,6$ & $-1,4$ & $-3,4$ \\
\hline & 1980-1989 & $-3,1$ & $-5,9$ & 10 & 1,5 & $-14,7$ \\
\hline & 1990-1999 & 2,3 & 0,9 & 20,3 & 4,3 & $-8,6$ \\
\hline & 2000-2009 & 4,2 & $-1,3$ & 12,1 & $-0,1$ & 15,5 \\
\hline & 2010-2017 & -1 & 1,9 & $-3,1$ & -1 & 0,2 \\
\hline \multirow{9}{*}{ Débits } & 1930-1939 & 8,8 & 8,2 & 17,4 & $-1,5$ & 13,6 \\
\hline & 1940-1949 & 14,9 & 17,7 & 17,3 & 12,7 & 12,7 \\
\hline & 1950-1959 & $-5,1$ & $-2,4$ & $-8,8$ & $-4,5$ & $-6,3$ \\
\hline & 1960-1969 & 5,9 & 11,3 & 4,3 & 1,9 & 4,9 \\
\hline & 1970-1979 & $-4,6$ & $-3,4$ & $-6,3$ & $-2,6$ & $-6,8$ \\
\hline & 1980-1988 & $-8,6$ & $-17,8$ & $-10,5$ & 6,1 & $-10,9$ \\
\hline & 1990 & - & - & - & - & - \\
\hline & 2001-2009 & 0,1 & $-3,2$ & -4 & 1,5 & 4,6 \\
\hline & 2010-2017 & $-13,2$ & $-11,6$ & $-15,1$ & $-14,2$ & $-13,1$ \\
\hline \multirow{9}{*}{ Débits de base } & 1930-1939 & 14,1 & 10 & 21 & 5,3 & 18,2 \\
\hline & 1940-1949 & 23,4 & 22,5 & 22,5 & 22,5 & 23,1 \\
\hline & 1950-1959 & $-6,6$ & $-11,9$ & $-7,1$ & $-3,3$ & $-4,8$ \\
\hline & 1960-1969 & 10,6 & 12,5 & 8,2 & 8,3 & 9,3 \\
\hline & 1970-1979 & 0,6 & 0,3 & $-1,2$ & 2,5 & $-1,3$ \\
\hline & 1980-1988 & $-33,5$ & -26 & $-22,3$ & $-11,6$ & $-22,8$ \\
\hline & 1990 & - & - & - & - & - \\
\hline & 2001-2009 & 6,5 & 2,5 & $-1,4$ & 9,6 & 11 \\
\hline & 2010-2017 & $-10,4$ & $-10,1$ & $-10,1$ & $-14,4$ & -10 \\
\hline \multirow{8}{*}{$\mathrm{Ke}$} & 1940-1949 & 7,5 & 20,9 & $-15,9$ & 17,1 & 19,8 \\
\hline & 1950-1959 & 11,6 & $-2,7$ & 39,2 & $-6,3$ & $-1,6$ \\
\hline & 1960-1969 & 13,6 & 3,1 & 42,7 & $-3,4$ & $-4,7$ \\
\hline & 1970-1979 & $-3,7$ & $-2,3$ & $-5,8$ & $-5,2$ & -2 \\
\hline & 1980-1988 & $-8,5$ & $-12,7$ & $-24,5$ & 2,4 & 6,8 \\
\hline & 1990 & - & - & - & - & - \\
\hline & 2001-2009 & $-7,6$ & 1,3 & $-18,6$ & 7,3 & $-9,5$ \\
\hline & 2010-2017 & $-12,4$ & $-8,4$ & $-17,8$ & $-14,2$ & $-8,9$ \\
\hline
\end{tabular}

lioration de la pluviométrie des décennies 1990 et 2000 sur le bassin n'a pas pu entraîner leur reconstitution. C'est ce qui a provoqué des disproportions parfois importantes entre les diminutions de la pluviométrie et de l'écoulement à partir de la décennie 1970.

Durant le printemps, les précipitations ont évolué modérément, de sorte qu'aucune rupture n'a été détectée dans leur série. A l'inverse, les débits de base, les débits moyens et les coefficients d'écoulement de cette saison diminuent de manière statistiquement significative depuis la décennie 1970. De même, l'examen des écarts à la moyenne de ces variables montre généralement des diminutions importantes pour ces mêmes variables, pendant que les pluies ne diminuent pas ou diminuent peu (Tableau 1). Le fléchissement des pluies d'hiver depuis la décennie 1970 semble être en rapport étroit avec celui des écoulements du printemps. Le rythme d'évolutions de ces deux variables au fil des décennies est similaire. Cela est clairement visible au cours de la décennie 2000 pour laquelle, une hausse des pluies d'hiver a entraîné un relèvement considérable des débits de base, des coefficients d'écoulement et des débits du printemps (Tableau 1). Il en est de même au cours de la décennie d'après où un fléchissement des pluies d'hiver est synchrone avec celui des variables d'écoulement étudiées, pendant que les pluies du printemps ont diminué elles-mêmes d'environ $2 \%$. Cette influence des précipitations d'hiver a déjà été suggérée par Liénou et al. (2008). Ces auteurs soutiennent qu'à partir de la décennie 1970, la baisse des pluies d'hiver crée 
un plus important déficit hydrique (évaporation et réserve en eau du sol) du bassin versant au début de la première saison des pluies du printemps. Cette aridification de l'hiver fait qu'une partie plus importante des précipitations reçues pendant le printemps participe d'abord au comblement de ce déficit hydrique, et donc la fraction qui génère effectivement les écoulements se trouve réduite. Ce qui se traduit alors par des lames écoulées plus faibles pour les mêmes hauteurs moyennes de précipitations durant le printemps, c'est ce qui explique la baisse du coefficient d'écoulement.

En automne, les pluies et les écoulements diminuent très légèrement sur l'ensemble de la période d'étude. Mais cependant, au cours des décennies 1980 et 2000, les variables d'écoulement augmentent, alors que les pluies ne varient pas beaucoup dans l'ensemble. Cette augmentation résulterait de la hausse des pluies d'été. L'impact des précipitations d'été sur les écoulements d'automne est davantage perceptible durant les décennies 2010 pour laquelle il est noté une diminution conjointe des pluies d'été, des débits de base, des coefficients d'écoulement et des débits d'automne, malgré une variation négligeable des pluies d'automne (Tableau 1). Au regard de ces évolutions, il ressort que, l'augmentation des pluies au cours de la saison sèche d'été durant les décennies allant de 1970-1990, réduit considérablement le déficit d'évaporation et des réserves en eau du sol au début de la saison de pluies d'automne, favorisant le ruissellement (Liénou et al., 2008; Ebodé et al., 2020). La partie des précipitations qui participe effectivement à l'écoulement s'accroît, d'où une augmentation du coefficient d'écoulement d'automne au cours de ces décennies (Tableau 1). La diminution des pluies d'été durant les décennies 2000 et 2010 entraîne l'effet inverse, c'est pourquoi il est constaté au cours de ces dernières une réduction des coefficients d'écoulement, responsable d'une baisse des débits.

\section{Conclusion}

Jusqu'à présent, peu d'études d'hydroclimatologie ont porté sur le bassin de l'Ogooué à Lambaréné, du fait des lacunes d'observation dans les séries chronologiques des débits et de l'inexistence des données de pluies dans certaines parties du bassin après les années 2000. Pour effectuer cette étude, nous avons réalisé un travail de validation de deux sources de données pertinentes disponibles pour la région (SIEREM et TRMM). Un réajustement (au moyen des coefficients correcteurs) des données TRMM a par la suite été opéré pour les mois présentant des écarts importants entre les deux sources. La série complète des pluies obtenue explique assez bien la variabilité des écoulements sur ce bassin. L'analyse de cette série des précipitations et celle des écoulements montre en effet une évolution synchrone entre les deux variables comparées. En plus de la concordance notée entre elles à l'échelle annuelle au fil du temps, on constate également que les décennies 1970 et 1980, marquent le début d'un changement important dans l'évolution des précipitations des saisons sèches dans le bassin, caractérisé par une augmentation des volumes précipités en été et une diminution en hiver. Ces modifications observées jusqu'à la fin de la décennie 1990 ont provoqué une modification des écoulements des saisons de pluies qui les suivent directement. Il a ainsi été relevé une augmentation des écoulements d'automne et une baisse de ceux d'hiver sur cette même période. Le début de la décennie 2000 est marqué par une inversion des tendances. On note désormais une baisse des pluies d'été accompagnée d'une diminution des écoulements d'automne, et inversement une hausse de celles d'hiver synchrone avec un relèvement des écoulements du printemps.

Disponibilité du code. Les logiciels utilisés pour l'analyse et le traitement des données dans cette étude (Khronostat 1.01 et QGIS 3.20) sont gratuits. Les liens donnant accès aux sites sur lesquels ils peuvent être téléchargés sont les suivants : http://www. hydrosciences.org/index.php/2020/09/04/khronostat/ (pour le premier) (HydroSciences Montpellier, 2021) et https://www.qgis.org/ fr/site/forusers/download.html (pour le second) (Open Source Geospatial, 2021).

Disponibilité des données. Les données de débits utilisées dans cette étude proviennent du SIEREM. Pour entrer en possession de ces données, il faudrait nécessairement entrer en contact avec la responsable actuelle de la banque des données à l'adresse : nathalie.rouche@umontpellier.fr. Les données de pluies (SIEREM et TRMM) par contre, sont disponibles gratuitement en ligne sur les sites : http://www.hydrosciences.fr/sierem/ (HydroSciences Montpellier, 2019) et https://giovanni.gsfc.nasa.gov (NASA, 2019).

Collaborateurs. Ce travail a été conceptualisé par VBE. La collecte, le traitement et l'analyse des données ont été effectués par VBE. Le projet a été dirigé par GM. La rédaction du manuscrit et la relecture ont été faites par VBE, EA et GM.

Intérêts concurrents. Les auteurs déclarent qu'ils n'ont aucun conflit d'intérêts.

Clause de non-responsabilité. Publisher's note : Copernicus Publications remains neutral with regard to jurisdictional claims in published maps and institutional affiliations.

Déclaration du numéro spécial. This article is part of the special issue "Hydrology of Large River Basins of Africa". It is a result of the 4th International Conference on the "Hydrology of the Great Rivers of Africa", Cotonou, Benin, 13-20 November 2021. 
Remerciements. Les auteurs remercient chaleureusement les agents de la Représentation de l'IRD (Institut de Recherche pour le Développement) au Cameroun et la direction du LMI DYCOFAC (notamment Jean-Jacques Braun), à Yaoundé, pour leur soutien administratif.

\section{Références}

Alhassane, A., Salack, S., Ly, M., Lona, I., Traore, S. B., et Sarr, B. : Evolution des risques agroclimatiques associés aux tendances récentes du régime pluviométrique en Afrique de l'Ouest soudano-sahélienne, Sécheresse, 24, 282-93, https://doi.org/10.1684/sec.2013.0400, 2013.

Bogning, S., Frappart, F., Paris, A., Blarel, F., Ninõ, F., Picart, S. S., Lanet, P., Seyler, F., Mahé, G., Onguene, R., Bricquet, J. P., Etame, J., Paiz, M. C., and Braun, J. J. : Hydroclimatology study of the Ogooué River basin using hydrological modeling and satellite altimetry, Adv. Space Res., 68, 672-690, https://doi.org/10.1016/j.asr.2020.03.045, 2020.

Buisson, A. : La grande saison sèche 1985 au Gabon : Situation climatique en Afrique intertropicale, La Météorologie, 8 , 5-13, 1985a.

Buisson, A. : Une anomalie climatique au Gabon en 1984, La Météorologie, 7, 36-47, 1985b.

Carré, P. : Fleuves et rivières du Gabon. Diversité des régimes hydrologiques, République gabonaise, Centre national de la recherche scientifique et technologique, Section d'études et recherches en hydrologie, ORSTOM, Libreville, Gabon, 22 pp., 1978.

Conway, D. P., Persechino, A., Ardoin-Bardin, S., Hamandawana, H., Dieulin, C., and Mahé, G. : Rainfall and river flow variability in sub-saharan Africa during the 20th century, J. Hydrometeorol., 10, 41-59, 2009.

Descroix, L., Sané, Y., Mamadou, T., Manga, S-P., Boubacar, D., Mingou, J., Mendy, V., Coly, S., Dièye, A., Badiane, A., Senghor, M.-J., Diedhiou, A.-B., Djiby, S., Bouaita, Y., Soumaré, S., Diop, A., Bakary, F., Bamol, A., Machu, E., Montoroi, J.-P., Andrieu, J., and Vandervaere, J.-P. : Inverse estuaries in West Africa : Evidence of the rainfall recovery ?, Water, 12, 647, https://doi.org/10.3390/w12030647, 2020.

Dieulin, C., Mahé, G., Paturel, J. E., Ejjiyar, S., Tramblay, Y., Rouché, N., and Mansouri, B. E. : A new 60-year monthly-gridded rainfall data set for Africa, Water, 11, 387, https://doi.org/10.3390/w11020387, 2019.

Ebodé, V. B. : Variabilité hydroclimatique en Afrique centrale occidentale forestière : entre analyse des fluctuations observées, recherche des facteurs explicatifs et modélisation prédictive, Generis Publishing, Miron Costin, République de Moldavie, 382 pp., 2020.
Ebodé, V. B., Mahé, G., Dzana, J. G., and Amougou, J. A. : Anthropization and climate change : impact on the discharges of forest watersheds in Central Africa, Water, 12, 2718, https://doi.org/10.3390/w12102718, 2020.

Electricité de France (E. D. F.) : Prospection hydro-électrique générale des bassins de l'Ogooué et de la Nyanga, Rapport intérimaire de la première campagne d'étude, Ministère des travaux publics, Libreville, République Gabonaise, 103 pp., 1961.

HydroSciences Montpellier : Grilles de pluies, accessible à l'adresse suivante : http://www.hydrosciences.fr/sierem/, la date du dernier accès : 7 mai 2019.

HydroSciences Montpellier : Khronostat, accessible à l'adresse suivante : http://www.hydrosciences.org/index.php/2020/09/04/ khronostat/, la date du dernier accès : 20 octobre 2021.

Liénou, G., Mahé, G., Paturel, J.-E., Servat, E., Sighomnou, D., Ekodeck, G. E., Dezetter, A., et Dieulin, C. : Evolution des régimes hydrologiques en région équatoriale camerounaise : un impact de la variabilité climatique en zone équatoriale, Journal des Sciences Hydrologiques, 53, 789-800, 2008.

Lodoun, T., Giannini, A., Traore, P. S., Some, L., Moussa, S., Vaksmann, M., and Rasolodimby, J. M. : Changes in seasonal descriptors of precipitation in Burkina Faso associated with late 20th century drought and recovery in West Africa, Environmental Development, 5, 96-108, https://doi.org/10.1016/j.envdev.2012.11.010, 2013.

Lubès, H., Masson, J. M., Servat, E., Paturel, J. E., Kouame, B., et Boyer, J. F. : Caractérisation des fluctuations dans une série chronologique par applications de tests statistiques, Etudes bibliographiques UR2-CEC, Orstom, Montpellier, France, 1994.

Mahé, G., Lerique, J., et Olivry J. C. : L'Ogooué au Gabon : Reconstitution des débits manquants et mise en évidence de variations climatiques à l'équateur, Hydrologie Continentale, 5, 105-124, 1990.

Mahé, G., Liénou, G., Descroix, L., Bamba, F., Paturel, J.-E., Laraque, A., Meddi, M., Habaieb, H., Adegea, O., Dieulin, C., Chahnez, K. F., and Khomsi, K. : The rivers of Africa : witness of climate change and the human impact on the environment, Hydrol. Process., 27, 2105-2114, 2013.

NASA : Earth Data, NASA, accessible à l'adresse suivante : https: //giovanni.gsfc.nasa.gov, la date du dernier accès : 11 mai 2019.

Open source Geospatial : QGIS, accessible à l'adresse suivante : https://www.qgis.org/fr/site/forusers/download.html, la date du dernier accès : 22 octobre 2021.

Ozer, P., Hountondji, Y.-C., et Laminou, M. O. : Evolution des caractéristiques pluviométriques dans l'est du Niger de 1940 à 2007, Geo-Eco-Trop., 33, 11-30, https://orbi.uliege.be/handle/ 2268/78267 (la date du dernier accès : 22 octobre 2021), 2009. 